\title{
Determination of the Broadband Aerosol Optical Depth Baseline and comparison with sunphotometer data
}

\section{Determinación de la Línea Base del Espesor Óptico de Aerosoles de Banda Ancha y comparación con datos de fotómetro solar}

\author{
F. García1 $1^{*}$, R. Estevan¹, J. C. Antuña-Marrero ${ }^{1}$, J. Rosas ${ }^{1}$, I. Y. Platero ${ }^{1}$, J. C. Antuña- \\ Sánchez ${ }^{1}$., N. Díaz ${ }^{1}$ \\ 1. Grupo de Óptica Atmosférica de Camagüey, Centro Meteorológico Provincial de Camagüey, Cuba \\ ${ }^{(*)}$ E-mail: frank@goac.cu
}

Received: 15/05/2015 Accepted: 06/11/2015

DOI: $10.7149 /$ OPA.48.4.249

\begin{abstract}
:
The Broadband Aerosol Optical Depth (BAOD) was calculated for Camagüey Actinometrical Station (EAC) using the methodology described by Gueymard in 1998. The main source of data used was the Solar Radiation Database of EAC for the 1981-2013 period. The BAOD calculation was performed for observations with total cloud coverage equal or less than one tenth of sky covered by clouds (Clear Sky, HCD) and for actinometrical observations with no clouds in the line of sight of the sun. To determine the BAOD Baseline the periods of El Chichón and Mt. Pinatubo volcanic eruptions were eliminated. The average value of BAOD for the entire period under aerosol background conditions is $0.115( \pm 0.075)$ with a decreasing trend of $-.20 \times 10^{-6}$ day $^{-1}$. BAOD values were compared with time coincident spectral AOD values obtained from a nearby sunphotometer. The highest correlation coefficient values were obtained for the wavelengths of 500 and $675 \mathrm{~nm}$, with an $\mathrm{R}^{2}=0.45$ for both cases.
\end{abstract}

Key words: aerosol optical depth, solar radiation, sunphotometry, actinometrical station, clear sky

\section{RESUMEN:}

El Espesor Óptico de Aerosoles de Banda Ancha (BAOD) fue calculado empleando, para la Estación Actinométrica de Camagüey (EAC), la metodología descrita por Gueymard en 1998. El BAOD fue obtenido utilizando como fuente de datos fundamental la Base de Datos de Radiación Solar de la EAC para el período 1981 - 2013. El cálculo del BAOD fue realizado para las observaciones con cobertura nubosa igual o inferior a 1 décimo de cielo cubierto y para las observaciones sin nubes en la línea de visión del instrumento. Para determinar la Línea Base fueron eliminados los períodos de las erupciones volcánicas de El Chichón y el Monte Pinatubo. El valor medio del BAOD para todo el período y para condiciones de fondo es de $0.115( \pm 0.075)$ con una tendencia decreciente igual a $-1.20 \times 10^{-6} \mathrm{dia}^{-1}$. Los valores del BAOD fueron comparados con valores de AOD espectral, coincidentes en tiempo, obtenidos del fotómetro solar ubicado en las cercanías de la estación. Los mayores valores del coeficiente de correlación fueron obtenidos para las longitudes de onda de 500 y $675 \mathrm{~nm}$, con un $\mathrm{R}^{2}=0.45$ para ambas.

Palabras clave: espesor óptico de aerosoles, radiación solar, fotometría solar, estación actinométrica, cielo despejado

\section{REFERENCES AND LINKS / REFERENCIAS Y ENLACES}

[1] IPCC, Climate Change 2013. The Physical Science Basis. Contribution of Working Group I to the Fifth Assessment Report of the Intergovernmental Panel on Climate Change [Stocker, T. F., et al. (eds.)]. Cambridge, United Kingdom and New York, Cambridge University Press (2013). 
[2] B. N. Holben, D. Tanre', A. Smirnov, T. F. Eck, I. Slutsker, N. Abuhassan, W. W. Newcomb, J. S. Schafer, B. Chatenet, F. Lavenu, Y. J. Kaufman, J. Vande Castle, A. Setzer, B. Markham, D. Clark, R. Frouin, R. Halthore, A. Karneli, N. T. O’Neill, C. Pietras, R. T. Pinker, K. Voss, G. Zibordi, "An emerging groundbased aerosol climatology: Aerosol optical depth from AERONET," J. Geophys. Res. 106, D11, 1206712097 (2001).

http://dx.doi.org/10.1029/2001JD900014

[3] A. Smirnov, B. N. Holben, T. F. Eck, O. Dubovik, I. Slutsker, "Cloud-Screening and Quality Control Algorithms for the AERONET Database," Rem. Sens. Env. 73, 337-349 (2000). http://dx.doi.org/10.1016/S0034-4257(00)00109-7

[4] C. A. Gueymard, "Turbidity Determination from Broadband Irradiance Measurements: A Detailed Multicoefficient Approach," J. Appl. Meteor. 37, 4, 414-435 (1998). http://dx.doi.org/10.1175/1520-0450(1998)037<0414:TDFBIM>2.0.C0;2

[5] A. Fonte, J. C. Antuña, "Caracterización del espesor óptico de banda ancha de los aerosoles troposféricos en Camagüey," Rev. Cub. Meteorol. 17, 1, 15-26 (2011).

[6] R. Estevan, J. C. Antuña, B. Barja, V. E. Cachorro, Á. M. de Frutos, A. Berjón, C. Toledano, B. Torres, R. Rodrígo, T. A. Hernández, C. E. Hernández, "Preliminary results of aerosols measurements with sun photometer at Camagüey, Cuba," Opt. Pura Apl. 44 (1), 99-106 (2011).

[7] J. C. Antuña, C. Hernández, R. Estevan, B. Barja, A. Fonte, T. Hernández, J. C. Antuña Jr, "Camagüey’s solar radiation rescued dataset: preliminary applications," Opt. Pura Apl. 44 (1) 43-48 (2011).

[8] SDRS para Cuba, http://www.goac.cu/actino (2015).

[9] GOAC, "Manual de Observaciones Actinométricas. Manual de procedimiento para Observadores Actinométricos," Grupo de Óptica Atmosférica de Camagüey, pp. 37 (2010).

[10] R. Estevan, Certificación de depósito legal facultativo de obras protegidas; software: "Actino 2.0"; CENDA 218-2010 (2010).

[11] J. C. Antuña, A. Fonte, R. Estevan, B. Barja, R. Acea, J.C. Antuña, "Solar Radiation Data Rescue at Camagüey, Cuba," Bull. Amer. Meteor. Soc. 89, 1507-1511 (2008). http://dx.doi.org/10.1175/2008BAMS2368.1

[12] J. C. Antuña, R. Estevan, A. Fonte, B. Barja, R. Acea, C. E. Hernández, T. Cordero, "Rescate de datos actinométricos y servicio de diagnóstico de radiación solar en Camagüey," Informe Científico Técnico, Proyecto: Sistema integrado de observaciones de radiación solar en Cuba, Camagüey, INSMET, pp. 126 (2010).

[13] R. Estevan, J. C. Antuña, B. Barja, F. García, C. E. Hernández, T. Cordero, J. Rosas, I. Morejón, "Climatología de la Radiación Solar en Camagüey 1981 - 2010. Sistema integrado de observaciones de radiación solar en Cuba," Informe Científico Técnico, Proyecto: Sistema integrado de observaciones de radiación solar en Cuba, Camagüey, INSMET, pp. 41 (2012).

[14] GGO, Manual para la operación e instalación de instrumentos de radiación solar, Ed. Guidrometeoizdat, pp. 124 (En Ruso) (1957).

[15] GGO, Manual para la calibración de instrumentos meteorológicos, Ed. Guidrometeoizdat, pp. 124 (En Ruso) (1959).

[16] R. Estevan, L. Mona, N. Papagiannopoulos, J. C. Antuña, V. Cachorro, A. de Frutos, "CALIPSO and sunphotometer measurements of Saharan Dust events over Camagüey," Opt. Pura Apl. 47 (3), pp. 189-196, (2014) http://dx.doi.org/10.7149/OPA.47.3.189

[17] B. Barja, S. Mogo, V. E. Cachorro, J. C. Antuña, R. Estevan, A. Rodrigues, Á. de Frutos, "Atmospheric particulate matter levels, chemical composition and optical absorbing properties in Camagüey, Cuba," Environ. Sci.: Processes Impacts 15, 440-453, (2013) http://dx.doi.org/10.1039/C2EM30854A

[18] R. Haltore, T. F. Eck, B. N. Holben, B. L. Markham, "Sun photometric measurements of atmospheric water vapor column abundance in the 940-nm band," J. Geophys. Res. 102, D4, 4343-4352 (1997) http://dx.doi.org/10.1029/96]D03247

[19] O. Cuesta, M. González, A. Collazo, "Algunas Características de la Deposición del Nitrógeno Atmosférico en Cuba," Revista Ciencias Ambientales 12, 47-53 (1996).

[20] V. A. Marén, "Caracterización de las concentraciones y dispersiones de los principales compuestos de nitrógeno atmosférico a Nivel Regional en Cuba," Trabajo de Diploma. InSTEC, Fac. de Gestión de la Ciencia, la Tecnología y el Medio Ambiente. Ciudad de La Habana, Cuba, pp. 124 (2009). 
[21] B. D. Santer BD, T. M. L. Wigley, J. S. Boyle, D. J. Gaffen, J. J. Hnilo, D. Nychka, D. E. Parker, K. E. Taylor, "Statistical significance of trends and trend differences in layer-average atmospheric temperature time series," Journal of Geophysical Research 105, 7337-7356 (2000). http://dx.doi.org/10.1029/1999JD901105

[22] P. R. Bevington, Data Reduction and Error Analysis for the Physical Sciences. New York , McGraw-Hill, 336 pp. (1969).

[23] B. Molineaux, P. Ineichen, N. O’Neill, "Equivalence of pyrheliometric and monochromatic aerosol optical depths at a single key wavelength," Applied Optics 37, 30, 7008-7018 (1998).

http://dx.doi.org/10.1029/1999JD901105

\section{Introducción}

La radiación solar es la principal fuente de energía del sistema Océano-Tierra-Atmósfera. Uno de los principales componentes de la atmósfera que interactúa con la radiación solar son los aerosoles, debido a su capacidad de absorberla y de dispersarla. El más reciente reporte del IPCC reconoce notables progresos científicos alcanzados en el conocimiento de la evolución espacio temporal de las concentraciones y de las propiedades ópticas de los aerosoles a escala global. También se ha logrado establecer que los aerosoles tienen efectos significativos en los procesos y fenómenos asociados al cambio climático, a la vez que pueden variar significativamente como resultado del mismo. Sin embargo, no hay consistencia en la magnitud y el signo de estos efectos, debido al limitado conocimiento de los procesos que rigen las fuentes de los aerosoles naturales y su compleja relación con el ciclo hidrológico sobre todo a escalas regionales y locales [1].

Uno de los principales parámetros que caracteriza las propiedades ópticas de los aerosoles es el Espesor Óptico de Aerosoles (AOD, por sus siglas en inglés). La Red Robótica de la NASA para la medición de Aerosoles (AERONET, por sus siglas en inglés) es una importante fuente de datos de este parámetro, que cuenta con una cantidad considerable de fotómetros solares a lo largo del planeta desde la década de los años 90 [2,3]. Esta técnica para la medición del AOD requiere de sofisticados y costosos instrumentos, así como, de calibraciones periódicas en sitios remotos con bajos valores de AOD, lo que crea dificultades a la hora de contar con una mejor distribución espacial del AOD a nivel global [4]. En 1998 Gueymard describió un método alternativo para la obtención del espesor óptico de aerosoles a partir de mediciones de radiación solar directa de banda ancha (BAOD) [4].

La Estación Actinométrica de Camagüey (EAC), cuenta con una larga serie de datos de radiación solar. Un trabajo anterior ha empleado las formulaciones descritas por Gueymard [4] para calcular el BAOD para la propia estación [5]. Una de las ventajas de realizar el estudio para la EAC es que en la misma se encuentra instalado un fotómetro solar CIMEL-318 desde finales de 2008 [6]. Esto posibilita la comparación de los valores de BAOD con valores de AOD espectral.

Para obtener la Línea Base del BAOD es necesario disponer de observaciones bajo condiciones de fondo. Teniendo esto en consideración se eliminaron de la base de datos los períodos con grandes perturbaciones, producidas por erupciones volcánicas. En el presente trabajo se detallan los procedimientos para la obtención de la Línea Base así como los resultados de la comparación de los valores del BAOD y el AOD a las diferentes longitudes de onda.

\section{Sitio de estudio, metodologías y bases de datos empleadas}

\section{2.a. Estación Actinométrica de Camagüey}

La Estación Actinométrica de Camagüey (EAC) se encuentra ubicada en los $21.42^{\circ} \mathrm{N}$ y $77.87^{\circ} \mathrm{W}$, a 118 metros sobre el nivel del mar. La EAC es del tipo Yanishevski, operada de forma manual por los observadores actinométricos y forma parte del Servicio Diagnóstico de Radiación Solar para Cuba [7,8]. Todos los sensores de radiación solar, utilizados en la EAC, son de banda ancha. El actinómetro empleado en la estación es del tipo M-3 y está acoplado a un galvanómetro del tipo GSA-1MA o GSA-1MB. Las observaciones son realizadas cada una hora desde la salida hasta la puesta del sol. El tiempo de duración de la observación puede llegar a ser de hasta 10 minutos. 
El proceso de toma de los datos es manual según lo establecido en el "Manual de Procedimientos para las Observaciones Actinométricas", registrándose las mismas en el libro de observaciones actinométricas. A continuación, empleando el software Actino 2.2, se procede a la creación del fichero digitizado de la observación y a su procesamiento de forma totalmente automatizada $[9,10]$.

En el 2007 se inició un proceso de rescate de datos de la EAC [11,12]. Durante este proceso se realizó además el rescate de la metadata de la estación y su digitalización, completamiento e introducción al software Actino $[9,10]$. En aquellos casos en que no se encontraron los pasaportes de los sensores y registradores que contienen los respectivos coeficientes de calibración, se procedió a su reconstrucción. Para ello se emplearon los libros de asentamiento de las observaciones actinométricas en los períodos correspondientes, en los que estaba establecido el registro de estos coeficientes para el cálculo manual [12]. En la actualidad los sensores son sujetos a calibraciones de campo cada 1 año.

\section{2.b. Base de datos de radiación solar}

La base de datos de radiación solar de la EAC versión 3.0, comprende un período de 33 años (1981-2013). Esta base de datos contiene observaciones horarias diurnas de los flujos de radiación solar en superficie. El período comprendido entre 1981 y 2010 fue empleado para obtener la climatología de la radiación solar en superficie para la EAC, para la cual los autores realizaron un exhaustivo proceso de control de calidad a la mencionada base de datos de ese período [13].

La versión 3.0 cuenta con mejoras en dos aspectos fundamentales en comparación con la empleada en el mencionado estudio realizado en Camagüey [5]. En primer lugar es más amplia que la versión 2.0 utilizada en ese estudio que cubría el período 1985 - 2007 (22 años). La versión 2.0 fue sometida al control de calidad según las metodologías establecidas para este tipo de estación [14,15]. En la versión 3.0 en adición a estos criterios, se tomaron en cuenta los criterios más modernos para el control de calidad de mediciones de radiación solar establecidos por la Red para la Línea Base de Radiación en Superficie (BSRN). Esos criterios incluyen pruebas de formatos y de consistencia de los datos. Entre estas se encuentran la consistencia interna y la tolerancia, las cuales son llevadas a cabo desde la introducción misma de los datos y realizadas nuevamente una vez que han sido procesados. Se incluyen las verificaciones de rango y la relación entre estos y las variables conexas, como es el caso de la temperatura seca, la húmeda y la humedad relativa. También se incluyen pruebas de consistencia temporal y de sumarización [13].

De las observaciones horarias se eliminaron las correspondientes a las 06:00 y 18:00 Hora Local (HL) para el cálculo del BAOD por introducir una mayor incertidumbre en el cálculo de las masas ópticas. Para garantizar que las observaciones sean representativas de las condiciones de fondo se eliminaron los períodos de grandes erupciones volcánicas. Tal es el caso de las erupciones de El Chichón en México (4/4/1982 al 4/3/1985) y el Monte Pinatubo (15/6/1991 al 15/5/1994).

Existen abundantes reportes de arribo de polvo Sahariano a la región del Caribe. En el caso particular de la EAC, sólo a partir del 2009, se han documentados eventos de este tipo para cuya determinación se emplearon mediciones de propiedades ópticas de los aerosoles obtenidas con fotómetro solar $[6,16]$ y de concentraciones de partículas empleando un impactador de partículas de bajo volumen [17]. Teniendo esto en consideración no se eliminaron estos datos dado que no constituye un criterio para toda la serie temporal al no disponer de reportes anteriores. No existe ningún otro evento en el área reportado en la bibliografía que afecte significativamente los valores de fondo del BAOD.

\section{2.c. Selección de Horarios para el Cálculo de BAOD}

Para determinar el BAOD con la mayor exactitud posible es necesario minimizar el efecto de la nubosidad sobre la radiación directa. Por ello se aplica el criterio de selección de los Horarios de Cielo Despejado (HCD) empleado anteriormente para el cálculo del BAOD en la EAC [5]. Éste criterio solo considera las observaciones con cobertura de nubes total igual o inferior a 1 décimo de cielo cubierto. Esto provoca el inconveniente de que quedan disponibles muy pocas observaciones para realizar el cálculo. En adición, no se garantiza que en el camino óptico no haya presencia de nubes, humo, polvo o bruma, afectando así la radiación directa. Según los procedimientos para la realización de las observaciones actinométricas [9], únicamente una observación en que el campo de visión del sol y $5^{\circ}$ alrededor de este, esté libre de nubes, humo, polvo y bruma (denominadas en actinometría Sol al Cuadrado - SC) garantiza eliminar este efecto. Luego de un análisis de cada uno de los criterios se determinó emplear las observaciones que cumplieran a la vez con ambos criterios (HCD + SC), lo cual constituye una novedad en los estudios que en este sentido 
se han realizado en Cuba. En la bibliografía consultada tampoco se encontraron trabajos de este tipo fuera de Cuba.

\section{2.d. Cálculo del BAOD}

Para el cálculo del BAOD se utilizó la siguiente ecuación [4]:

$$
B A O D=\left(\frac{1}{m_{a}}\right)\left[\ln \left(\frac{E_{0 n}}{E_{b n}}\right)-m_{R} \delta_{c}\right]-\delta_{w}-\delta_{n t}
$$

Donde:

$\mathrm{m}_{\mathrm{a}}$ : masa óptica de aerosoles,

$\mathrm{m}_{\mathrm{R}}$ : masa óptica de Rayleigh,

$E_{0 n}$ irradiancia medida en el tope de la atmósfera,

$E_{b n}$ : irradiancia directa medida en superficie,

$\delta_{c}$ : espesor óptico de la atmósfera limpia y seca,

$\delta_{\mathrm{w}}$ : espesor óptico del vapor de agua,

$\delta_{\text {nt: }}$ espesor óptico del $\mathrm{NO}_{2}$.

Las ecuaciones para la obtención de los espesores ópticos se detallan en el artículo [5]. A continuación se detallan los valores empleados para la obtención de cada uno de los componentes de la ecuación.

\section{2.e. Otros datos empleados para el cálculo del BAOD}

Para el cálculo de $\delta_{\mathrm{w}}$ se emplearon los valores medios del contenido integral de agua precipitable (WV) que para la estación están disponibles en AERONET, a la que tributa el fotómetro solar CIMEL-318 operado desde Octubre de 2008 por el Grupo de Óptica Atmosférica de Camagüey (GOAC). Los datos de WV son calculados a partir de las mediciones realizadas en la banda de $940 \mathrm{~nm}$ de este tipo de fotómetro para observaciones con una línea de visión, entre el instrumento y el sol, libre de nubes [9]. El error en los valores de WV de este instrumento es del $10 \%$ [18]. Se obtuvieron de AERONET las medias mensuales calculadas a partir de datos del nivel 2.0 de procesamiento. Esta base de datos es calculada luego de un proceso riguroso de control de calidad de las observaciones, que incluye además, los datos de pre y post calibración de cada instrumento, así como, un procesamiento para eliminar el efecto de la nubosidad [3].

El $\mathrm{NO}_{2}$ constituye otro de los compuestos incluidos en la expresión para al cálculo del BAOD. Existen varias referencias a concentraciones de $\mathrm{NO}_{2}$ troposférico en Cuba, fundamentalmente referentes a la capital del país [19,20]. En 2009 Maren [20] realizó un estudio al respecto para las localidades de Casablanca, Colón, Fallas, La Palma y Santiago de Cuba. El error en las concentraciones medidas no sobrepasó el 10 \%. Considerando las fuentes de origen de este contaminante, las condiciones del entorno y la cercanía geográfica, se tomaron como referencia los valores medios mensuales de $\mathrm{NO}_{2}$ troposférico en la localidad de Falla, la más representativa de las condiciones de la EAC. En el caso del $\mathrm{NO}_{2}$ estratosférico se empleó el valor de 0.000204 atm-cm referido en [5] tomado de los datos de la atmósfera estándar de Estados Unidos.

Para la obtención de $\delta_{c}$ se emplearon las concentraciones de ozono $\left(\mathrm{U}_{0}\right)$ referidas en [5]. Estos fueron obtenidos del sitio web del Total Ozone Mapping Spectrometer. Se obtuvieron los valores medios mensuales a partir de la información generada por este instrumento a bordo de los satélites Nimbus 7 (1978 - 1993) y Earth Probe (1996 - 2005).

La Tabla 1 muestra los valores de WV, Ozono y $\mathrm{NO}_{2}$ utilizados para el cálculo de cada uno de los respectivos valores de espesores ópticos de la ecuación 1 [5]. 


\begin{tabular}{|c|c|c|c|c|}
\hline Mes & $\mathrm{WV}(\mathrm{cm})$ & $\mathrm{U}_{0}(\mathrm{~atm}-\mathrm{cm})$ & $\begin{array}{c}\mathrm{NO}_{2} \\
\text { Troposférico } \\
\text { (atm-cm) } \\
\end{array}$ & $\begin{array}{c}\mathrm{NO}_{2} \\
\text { Estratosférico } \\
\text { (atm-cm) } \\
\end{array}$ \\
\hline ENE & 2.76 & 0.2489 & \multirow{12}{*}{$3.40 \times 10^{-4}$} & \multirow{12}{*}{$2.04 \times 10^{-4}$} \\
\hline FEB & 2.71 & 0.2567 & & \\
\hline MAR & 2.58 & 0.2682 & & \\
\hline $\mathrm{ABR}$ & 3.14 & 0.2860 & & \\
\hline MAY & 3.43 & 0.2948 & & \\
\hline JUN & 4.29 & 0.2946 & & \\
\hline JUL & 4.11 & 0.2947 & & \\
\hline AGO & 4.31 & 0.2891 & & \\
\hline SEP & 4.43 & 0.2812 & & \\
\hline OCT & 4.20 & 0.2692 & & \\
\hline NOV & 3.20 & 0.2563 & & \\
\hline DIC & 2.81 & 0.2512 & & \\
\hline
\end{tabular}

\section{2.f. Comparación con fotometría solar y obtención de la Línea Base}

Para la comparación entre los valores del BAOD y del AOD espectral, se emplearon los valores de este último parámetro obtenidos para las longitudes de onda de 340, 380, 440, 500, 675, 870, 1020 y $1640 \mathrm{~nm}$. Se determinaron las observaciones de BAOD y AOD espectral coincidentes en el intervalo de tiempo de una hora alrededor del tiempo medio de la observación actinométrica (30 minutos antes y 30 minutos después). Se encontraron un total de 715 observaciones actinométricas coincidentes con sus similares del fotómetro solar. Para cada caso se calcularon los valores medios del AOD para cada longitud de onda, así como, el valor medio del WV para el intervalo de tiempo mencionado. El BAOD fue calculado nuevamente para las observaciones coincidentes empleando el valor medio del WV antes mencionado.

Para determinar la Línea Base, se emplearon los valores del BAOD superiores a 0.002 que es el error del método de cálculo del BAOD empleado [4]. Se calcularon los principales estadígrafos para toda la muestra, así como, las tendencias y su significación estadística. La tendencia del BAOD para todo el periodo fue calculada a partir de un ajuste lineal por mínimos cuadrados. La significación estadística de dicha tendencia se determinó a partir de la prueba de t Student. El valor calculado de t se obtuvo mediante la siguiente ecuación [21]:

$$
t=\frac{b}{\sqrt{\frac{\sum\left(Y-Y^{\prime}\right)^{2}}{\sum(X-\bar{X})^{2}(N-2)}}}
$$

donde $\mathrm{b}$ es la pendiente de la recta y $\mathrm{N}$ los grados de libertad de la muestra.

\section{2.g. Error asociado al cálculo del BAOD}

Gueymard [4] describe las principales fuentes de error que tributan a la obtención del BAOD. Primeramente existe un error asociado al propio instrumento de medición, en este caso, el actinómetro. En el presente trabajo se ha considerado el mismo en el orden de un $5 \%$. Por otro lado, el WV constituye el elemento más sensible a tener en cuenta, pues es el de mayor peso en la ecuación para la obtención del BAOD. La incertidumbre de los valores de WV obtenidos por fotometría solar es cercana al $10 \%$ como se refiere con anterioridad. $\mathrm{El} \mathrm{NO}_{2}$ es el compuesto que contribuye en menor medida a la obtención del BAOD.

En el estudio referido anteriormente se empleó la aproximación de Bevington [22] para calcular el error final en la obtención del BAOD. Para el presente trabajo se calculó a través de la expresión dada por la ecuación 3 [4].

$$
\triangle B A O D=\left\{\left(m_{a}^{-1} \frac{\Delta E_{b n}}{E_{b n}}\right)^{2}+\left(\frac{m_{R}}{m_{a}}\right)^{2} \cdot\left[\left(\frac{d \delta_{c}}{d u_{0}} \Delta u_{0}\right)^{2}+\left(\frac{d \delta_{w}}{d w} \Delta w\right)^{2}\right]+\left(\frac{d \delta_{c}}{d u_{n t}} \Delta u_{n t}\right)\right\}^{\frac{1}{2}}
$$


Se asumieron márgenes de error del 10 y 20 \% para cada compuesto, así como la variación del WV desde 2 hasta $4.5 \mathrm{~cm}$. Se empleó para este cálculo un valor de $U_{0}=0.3 \mathrm{~atm}-\mathrm{cm}$. La Tabla 2 muestra los resultados obtenidos para ángulos cenitales de $10^{\circ}, 30^{\circ}, 60^{\circ}$ y $80^{\circ}$.

TABLA 2. Posible error en el cálculo del BAOD causado por $\pm 10 \%$ y $\pm 20 \%$ de error en las variables de entrada y para ángulos cenitales entre $10^{\circ}$ y $80^{\circ}$.

\begin{tabular}{|c|c|c|c|c|}
\hline \hline $\begin{array}{c}\text { WV } \\
(\mathrm{cm})\end{array}$ & 10 & 30 & 60 & 80 \\
\hline \multicolumn{1}{|c|}{ $\pm 10 \%$ de error } \\
\hline 2 & 0.04949 & 0.04355 & 0.02524 & 0.00896 \\
2.5 & 0.04953 & 0.04359 & 0.02528 & 0.00899 \\
3.5 & 0.04960 & 0.04366 & 0.02535 & 0.00904 \\
4.5 & 0.04967 & 0.04373 & 0.02541 & 0.00908 \\
\hline \multirow{2}{|}{ $\pm 20 \%$ de error } \\
\hline 2 & 0.05019 & 0.04424 & 0.02588 & 0.00950 \\
2.5 & 0.05035 & 0.04440 & 0.02603 & 0.00961 \\
3.5 & 0.05065 & 0.04469 & 0.02629 & 0.00980 \\
4.5 & 0.05091 & 0.04495 & 0.02652 & 0.00996 \\
\hline \hline
\end{tabular}

\section{Resultados y Discusión}

\section{3.a. Criterio de Selección de horarios para el cálculo del BAOD}

La Tabla 3 muestra la cantidad de observaciones para cada criterio de selección. El porciento de casos obtenidos para HCD concuerda con lo reportado para la región por otros autores [5]. El criterio de HCD es el más restrictivo de los tres. Sin embargo cuando se emplea de conjunto con las observaciones de SC $(\mathrm{HCD}+\mathrm{SC})$ resultan el mayor número de casos. Este último criterio representa un $3.18 \%$ más que el empleado con anterioridad (HCD). El criterio empleado para el cálculo del BAOD (HCD + SC) constituye una mejora con respecto al utilizado por trabajos anteriores para la misma estación [5] y permite contar con un número mayor de observaciones sin introducir el efecto de la nubosidad en la obtención del BAOD. Es de notar cuando se aplica el criterio HDC +SC el porciento de casos obtenidos es menor en aproximadamente un $2.3 \%$ que la suma de los porcientos individuales de los criterios HCD y SC. Este resultado demuestra que aun en presencia de $1 / 10$ de cielo cubierto en un $26 \%$ de los casos se registran nubes, humo, polvo o bruma en el campo de visión del actinómetro cuando se apunta al sol.

TABLA 3. Número de casos según criterio de nubosidad

\begin{tabular}{|c|c|c|}
\hline \hline Citerio & Observaciones & \% del Total \\
\hline \hline HCD & 3317 & 3.32 \\
SC & 5460 & 5.46 \\
HCD + SC & 6497 & 6.50 \\
\hline \hline
\end{tabular}

\section{3.b. Estadígrafos generales y tendencia para todo el período}

En la Tabla 4 se aprecia la estadística general del BAOD así como de los espesores ópticos para los otros componentes descritos en la Ecuación 1. Para todo el período se encontró un valor mínimo absoluto del BAOD de $0.25 \times 10^{-2}$, ocurrido el 17 de marzo de 1996 y un valor máximo del BAOD de 0.922, ocurrido el 26 de marzo de 1995. El valor medio obtenido del BAOD fue de 0.115 ( \pm 0.075$)$. Se observa que el valor medio del $\delta_{\text {nt }}$ es tres órdenes de magnitud menor que el del BAOD a la vez que el valor medio de $\delta_{\mathrm{c}}$ es un orden de magnitud menor el del BAOD. Sin embargo el valor medio del $\delta_{\mathrm{w}} \mathrm{y}$ del BAOD es del mismo orden. Por esta razón es que la determinación precisa del WV desempeña un importante papel en la determinación del BAOD.

TABLA 4. Espesor óptico de los principales componentes de la ecuación 1: $\delta_{c}$ : espesor óptico de la atmósfera limpia y seca; $\delta_{\text {w: }}$ espesor óptico del vapor de agua y $\delta_{\text {nt: }}$ espesor óptico del $\mathrm{NO}_{2}$.

\begin{tabular}{|c|c|c|c|c|}
\hline \hline & Máximo & Mínimo & Media & Desv. Estándar \\
\hline \hline BAOD & $9.22 \times 10^{-1}$ & $0.25 \times 10^{-2}$ & $1.15 \times 10^{-1}$ & $7.47 \times 10^{-2}$ \\
$\delta_{\mathrm{c}}$ & $1.18 \times 10^{-1}$ & $4.25 \times 10^{-2}$ & $9.97 \times 10^{-2}$ & $1.31 \times 10^{-2}$ \\
$\delta_{\mathrm{nt}}$ & $9.75 \times 10^{-4}$ & $6.09 \times 10^{-4}$ & $9.55 \times 10^{-4}$ & $3.62 \times 10^{-5}$ \\
$\delta_{\mathrm{w}}$ & $1.84 \times 10^{-1}$ & $2.69 \times 10^{-2}$ & $1.17 \times 10^{-1}$ & $3.15 \times 10^{-2}$ \\
\hline \hline
\end{tabular}


En la Figura 1 se muestra la serie del BAOD para todo el período, calculado para las observaciones que cumplen con el criterio HCD + SC. Los intervalos en que no aparecen valores del BAOD en la serie de datos corresponden a los períodos de las erupciones volcánicas y a períodos con falta de datos. La tendencia encontrada de $-1.20 \times 10^{-6} \mathrm{dia}^{-1}\left(-4.36 \times 10^{-4}\right.$ año $\left.{ }^{-1}\right)$, significativa al $99.9 \%$, concuerda con la tendencia positiva del Índice de Claridad reportada por Estevan et al. [13], para el período 1981-2010, así como, con la tendencia del BAOD referida anteriormente [5], calculada tomando solo en cuenta los HCD.

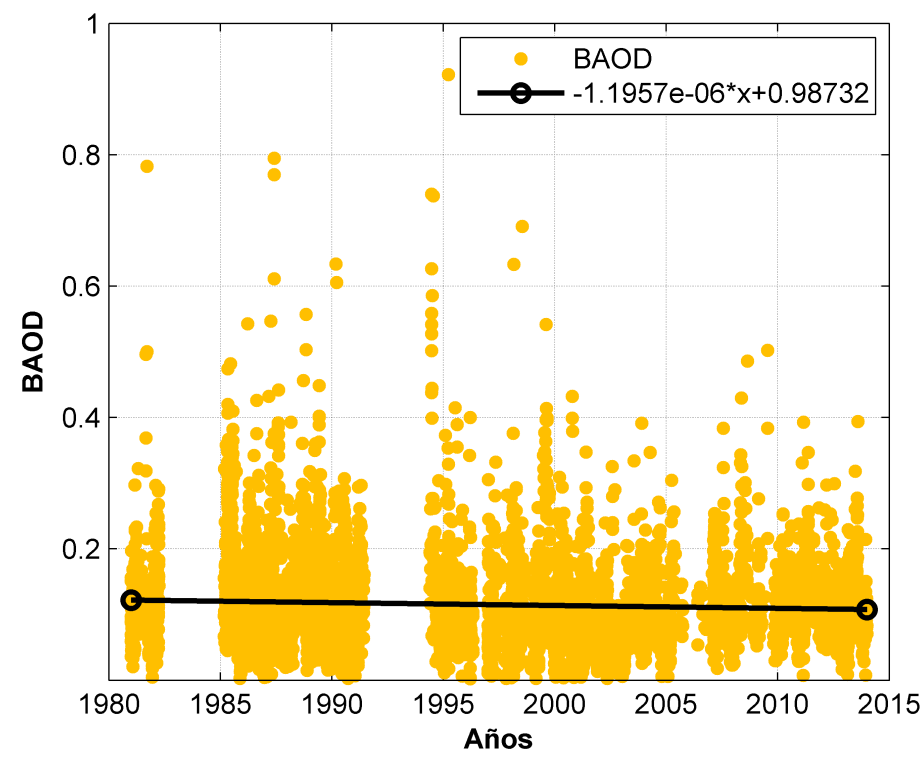

Fig. 1. Espesor Óptico de Banda ancha para todo el período, tomando en consideración las observaciones según el criterio HCD + SC de la Tabla 3.

El histograma de frecuencia (Fig. 2) muestra que la mayor cantidad de valores del BAO estuvieron centrados en valores entre 0.05 y 0.15 con más del $60 \%$ de la muestra. La frecuencia acumulativa del BAOD desde 0 hasta 0.15 es del $77.6 \%$ y se incrementa hasta casi el $95 \%$ cuando se toman los valores del BAOD hasta 0.25 . Solo un $3.6 \%$ de los casos reportados del BAOD se encuentran entre 2.5 y 0.35 y para valores del BAOD superiores los casos apenas rebasan el $1.4 \%$ de toda la muestra.

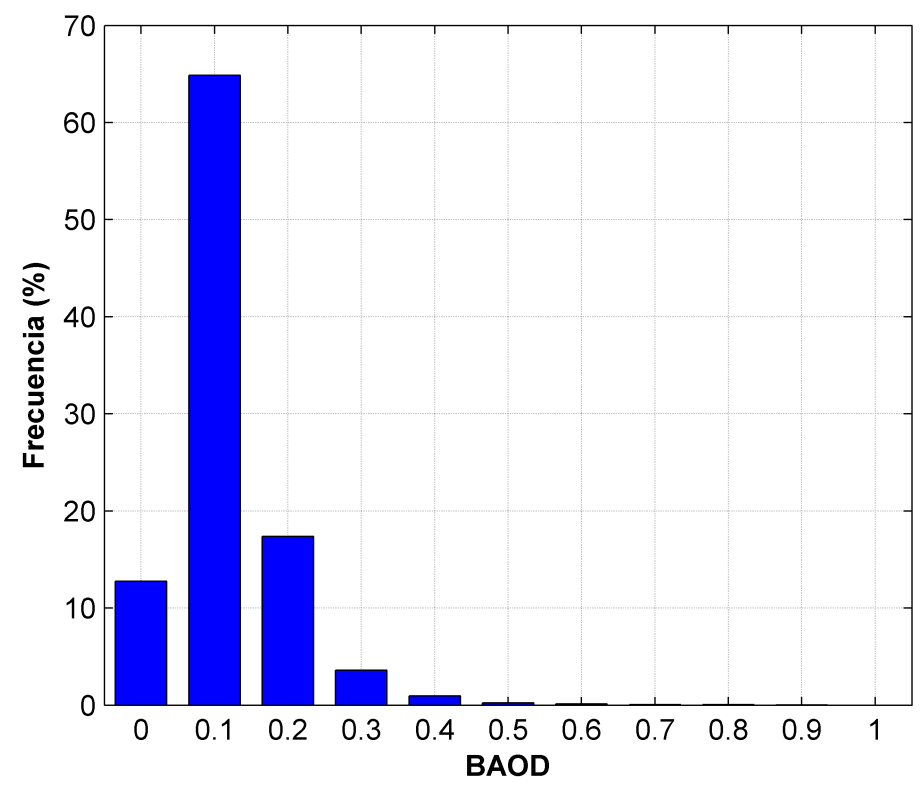

Fig. 2. Frecuencia de valores de BAOD para Camagüey 
El curso anual del BAOD se aprecia en la Fig. 3 que muestra los máximos agrupados en los meses de verano, junio, julio y agosto. Esto es consistente con la presencia en esos meses de los eventos de polvo sahariano en la región del Caribe reportado por varios autores [6,16].

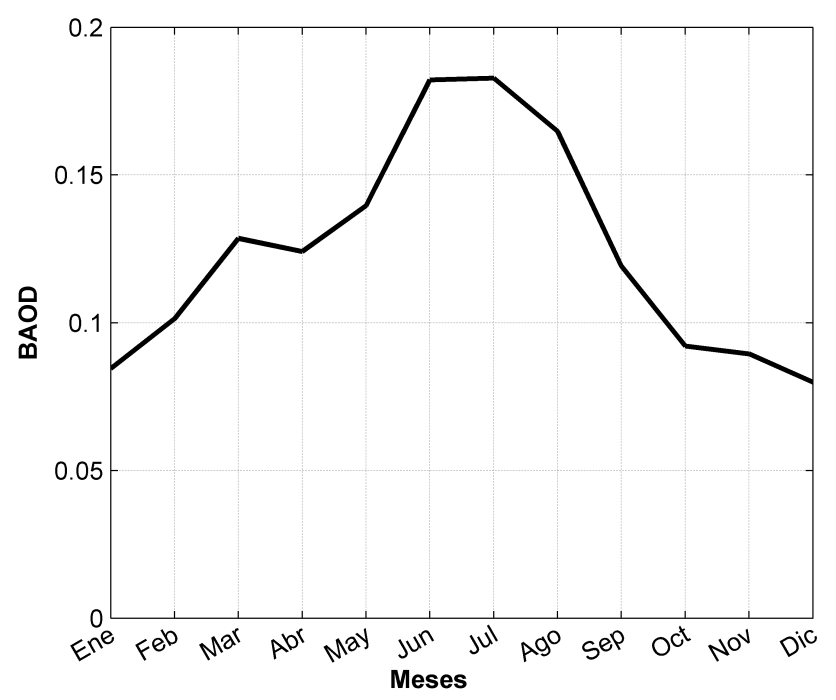

Fig. 3. Medias Mensuales del BAOD para todo el período 1981-2013

El curso diurno del BAOD no presenta grandes variaciones. Entre las 07 y 10 HL hay una ligera tendencia al crecimiento para luego decrecer hasta encontrar el valor mínimo a las $13 \mathrm{HL}$ con 0.109. Posteriormente ocurre un nuevo ascenso hasta arribar al valor máximo medio horario a las 16 HL con 0.126.

\section{3.c. Comparación con datos del fotómetro solar}

Los resultados de la comparación con el AOD de cada uno de las longitudes de onda medidas con el fotómetro solar mostraron mejores resultados cuando se emplearon los valores medios mensuales de WV que cuando se emplearon los valores medios horarios de WV. Las mejores correlaciones se obtuvieron para las comparaciones con las longitudes de onda de 675 y $500 \mathrm{~nm}$ con un mismo valor de $\mathrm{R}^{2}=0.45$ en ambos casos. Esto último concuerda con el trabajo de Molineaux et al. [23] en el que se determinó que 675 $\mathrm{nm}$ es la mejor longitud de onda para realizar la comparación con espesor óptico de banda ancha. Las menores diferencias porcentuales se hallaron en las longitudes de onda de 440 y $500 \mathrm{~nm}$ con valores promedio de $31.10 \%$ y $31.64 \%$ respectivamente.

También se realizó una comparación con las medias mensuales del BAOD y las correspondientes del AOD en 500 y 675 nm (Fig. 4). Para esta comparación se calcularon las medias mensuales del BAOD para el período 2008 - 2013, etapa que coincide con el período de datos del fotómetro en el sitio de estudio. Los meses de enero, noviembre y diciembre muestran menores diferencias porcentuales para el caso del AOD en $500 \mathrm{~nm}$. En el resto de los meses estas diferencias son menores para el AOD en $675 \mathrm{~nm}$.

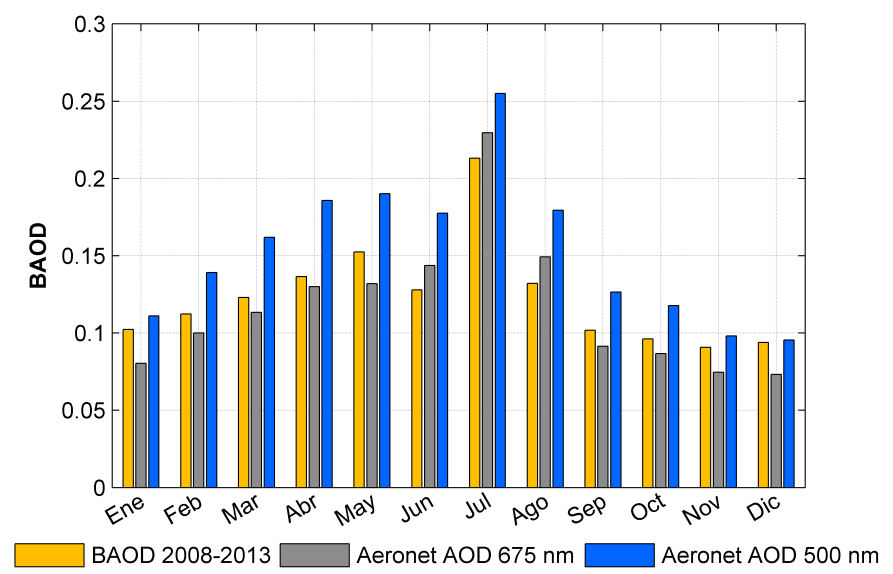

Fig. 4. Medias mensuales del BAOD para el período 2008 - 2013 y medias mensuales del AOD en 675 y $500 \mathrm{~nm}$. 


\section{Conclusiones}

La aplicación de un criterio más amplio y consistente para la selección de los horarios empleados en el cálculo del BAOD permitió obtener una mayor cantidad de casos y por consiguiente, ampliar la serie de datos para el cálculo del BAOD para la EAC. El empleo de este criterio permitió además elevar la calidad de las mediciones empleadas para el cálculo del BAOD al excluir los casos en que bajo condiciones de HCD existían nubes, humo, polvo o bruma en el campo de visión del actinómetro cuando este apuntaba al sol. El incremento del número de observaciones empleadas para el cálculo permitió obtener una estadística más robusta del BAOD. Se determinó la Línea Base del BAOD en la EAC para todo el periodo, con un valor medio de 0.115 ( \pm 0.075 ), un máximo de 0.922 y un mínimo de $0.2 \times 10^{-2}$.

La tendencia del BAOD para el periodo de 33 años, comprendidos entre 1981 y 2013 fue negativa, con un valor de $-1.20 \times 10^{-6} \mathrm{dia}^{-1}\left(-4.36 \times 10^{-4}\right.$ año $\left.{ }^{-1}\right)$ y con una significación estadística del $99.9 \%$. Este resultado concuerda con las tendencias negativas halladas por otros autores en trabajos anteriores [5,18]. Mientras, el curso anual del BAOD mostró un crecimiento del valor medio en los meses de verano, consistente con lo reportado en la bibliografía sobre el tema.

La comparación hecha con el AOD espectral, medido con fotómetro solar, mostró mejores resultados para las longitudes de onda de 500 y $675 \mathrm{~nm}$, con las mejores correlaciones para ésta última longitud de onda. Los resultaron de la comparación fueron mejores cuando se emplearon los valores medios mensuales del WV para el cálculo del BAOD que cuando se emplearon los valores medios horarios del WV.

\section{Agradecimientos}

Los autores expresan su más profundo agradecimiento al Dr. Ángel de Frutos Baraja y la Dra. Victoria E. Cachorro Revilla por sus esfuerzos en el establecimiento y mantenimiento del Sitio Camagüey en RIMA, contribuyendo además a AERONET. Este agradecimiento se hace extensivo al Grupo de Óptica Atmosférica de la Universidad de Valladolid por el apoyo y logística. 